\title{
Study of cirrus clouds and implications for the variability of laser guide star intensity and fratricide effects
}

\author{
Angel Otarola ${ }^{\mathrm{a}}$ and Paul Hickson ${ }^{\mathrm{b}}$ \\ aTMT International Observatory, 100 West Walnut Street, Suite 300, Pasadena, CA, USA \\ 91124 \\ ${ }^{\mathrm{b}}$ Dept. of Physics and Astronomy, University of British Columbia, 6224 Agricultural Road, \\ Vancouver, BC V6T 1Z1, Canada
}

\begin{abstract}
Measurements of back-scattering, at a wavelength of $532 \mathrm{~nm}$, from the Cloud-Aerosol Lidar and Infrared Pathfinder Satellite Observation (CALIPSO) satellite, over the period June 2006 - November 2016, are analyzed to characterize cirrus clouds at the location of several observatory sites worldwide. From these data, we derive statistics of altitude, thickness and optical depth of cirrus clouds. These parameters are relevant for understanding and modeling the effects of cloud and aerosols on laser propagation for adaptive optics and the temporal variability of the fratricide pattern. The latter is particularly important for facilities employing center-launched lasers, such as the Thirty Meter Telescope.
\end{abstract}

Keywords: Cirrus clouds, aerosols, Rayleigh backscattering, fratricide effect

\section{INTRODUCTION}

\subsection{Motivation}

Cirrus clouds impact the performance of adaptive optics in several ways. They attenuate the uplink laser beam, and also the downward propagating light, reducing laser guide star (LGS) brightness. They also attenuate the light from the science target, and may increase the diffuse background light that is the primary source of noise for observations of faint objects. Laser light scattered by the clouds contributes to fratricide, the contamination of LGS light by scattered light from multiple propagating laser beams.

A new generation of large aperture telescopes will make extensive use of adaptive optics employing asterisms created by multiple lasers. In the case of the Thirty Meter Telescope (TMT) project, it is planned that for first light at least $50 \%$ of the time will be dedicated to near-infrared diffraction limited imaging and spectroscopy. Consequently, this study focused on determining the physical characteristics (thickness and optical depth) as well as the statistical occurrence of thin cirrus clouds and aerosols at various astronomical sites. Most of those sites have telescopes that make use of adaptive optics systems employing LGS. The information learned in this study should prove helpful for simulations of LGS magnitude variability, as well as of variability in the intensity of the backscattered light detected by wavefront sensors.

\subsection{Background information}

Cirrus clouds are a source of attenuation of star light during astronomical observations. The fraction of light scattered out of the line of sight to the source depends on the optical density of the cirrus clouds. There is not much discussion in the literature about systematic detection and classification of cirrus clouds at astronomical observatory sites. Yet, even thin cirrus clouds are enough to perturb the quality of astronomical observations. ${ }^{1,2}$ In fact, isolated emissions features detected on low resolution spectroscopy observations, have been claimed to arise from light from distant lightning storms scattered by thin cirrus clouds. ${ }^{3}$ Of particular interest are thin cirrus clouds that contribute to transparency variations in the order of $10 \%$ to $20 \%$, i.e. variations in the detected star magnitude of order 0.1 to 0.2 magnitudes. ${ }^{4}$

Further author information: (Send correspondence to A.O.)

A.O.: E-mail: aotarola@tmt.org, Telephone: 15203188401 
Importantly, in the context of new large aperture telescopes as the European Extremely-Large Telescope $(\mathrm{ELT}),{ }^{5} \mathrm{TMT}^{6}$ and the Giant Magellan Telescope (GMT), ${ }^{7}$ which will use sodium LGS extensively, cirrus clouds will have a significant impact on performance. In telescopes employing adaptive optic (AO) systems using multiple center-launched lasers, such as the Gemini South $(\mathrm{GeMS})^{8}$ and the TMT (NFIRAOS) ${ }^{9}$ the backscattered laser light due to Rayleigh scattering from air molecules, and Mie scattering in cirrus clouds and from aerosol particles, is detected by the wavefront sensors matched to neighboring LGS. This is the so-called fratricide effect. ${ }^{10,11}$ The backscattered light is consequently a source of both photon noise and background variability and will ultimately affect the quality of the wavefront reconstructions. At the TMT project, there is a plan to compensate for the Rayleigh backscattering by making use of either a reference image of the Rayleigh backscattering pattern or by fitting a model to the fratricide pattern. ${ }^{12}$ However, the time variability of cirrus clouds optical depth, as the clouds drift across the propagating laser beams, will introduce variability in the intensity and shape of the fratricide pattern. Cirrus clouds will also reduce LGS brightness, reducing the number of detected photons and thus impacting the availability of the AO system. ${ }^{13}$

This manuscript includes the following sections: Section 2 explains the data used for this study, Section 3 summarizes the statistics of the various parameters of interest, Section 4 shows some calculations of laser light power attenuation and variability in the atmospheric channel, Section 5 presents some comments about the relevance of attenuation and variability in the cirrus cloud properties in the fluctuations of the laser fratricide pattern, and Section 6 summarizes relevant conclusions of this study.

\section{DATA}

The data used in this study were acquired by the Cloud-Aerosol Lidar with Orthogonal Polarization (CALIOP) instrument on board of the Cloud-Aerosol Lidar and Infrared Pathfinder Satellite Observations (CALIPSO) satellite. $^{14}$ These data were obtained from the NASA Langley Research Center Atmospheric Science Data Center*.

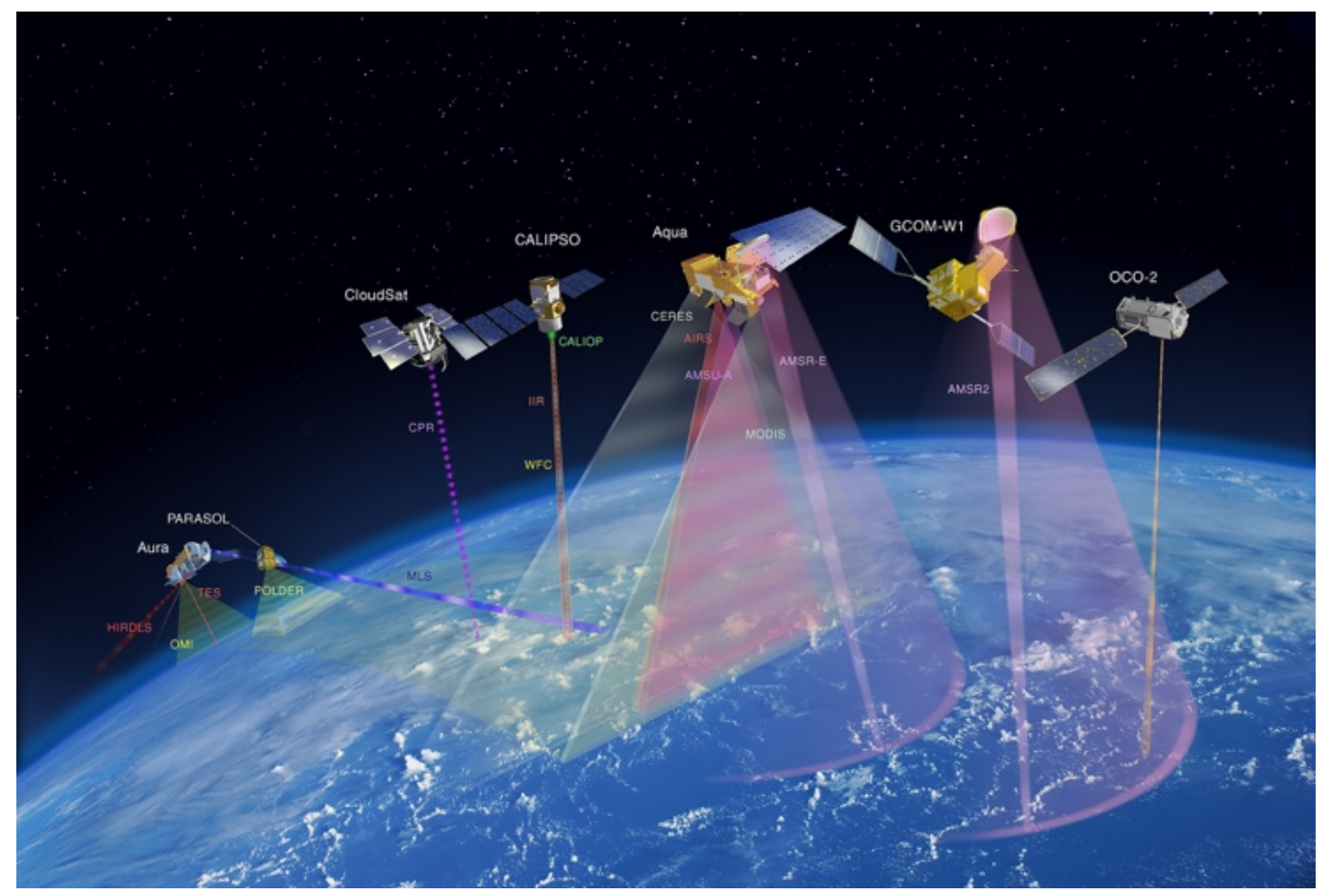

Figure 1. Artist concept of the constellation of satellites in the A-Train Orbit. Picture obtained from https://www.nasa.gov/content/about-the-a-train.

\footnotetext{
${ }^{*}$ https://www-calipso.larc.nasa.gov/search/index.php
} 
The CALIPSO satellite is part of a special Sun-synchronous orbit, so called the A-Train, which includes a convoy of satellites, each carrying complementary sensors for the study of various geophysical parameters, see Figure 1. The satellites are positioned so that the time elapsed from the passage of the first and last satellite over a given point along the orbit is only 15 minutes. The orbital height is $705 \mathrm{~km}$ and the satellites cross the equator at approximately 1:30 PM solar time, and then again around 1:30 AM. There are 14.55 orbits per day, this imply the satellites cover sections of the planet separated about 24.74 degrees each day. The orbit path, at the equator, progresses westwards 10.8 degrees each day, and the final coverage pattern results in a total of 233 orbits circling the planet with a separation of about 1.545 degrees. At the Equator, a separation of 1.545 degrees is about $172 \mathrm{~km}$.

CALIPSO includes an integrated LIDAR transmitter platform which in turn includes two LIDAR sensors (one using visible and the other a near-infrared wavelength), both in nadir-view geometry. An optical telescope of one meter diameter is used for the detection of the light backscattered, by aerosols and cloud particles, at various levels in the atmosphere as the laser light propagates from the satellite to the surface of the planet. Table 1 summarizes the main parameters of the LIDAR transmitter platform and detection system.

Table 1. CALIOP main parameters

\begin{tabular}{ll}
\hline Parameter & Value \\
\hline Laser & Nd:YAG, diode-pumped, Q-switched frequency doubled \\
Wavelength & $532 \mathrm{~nm}, 1064 \mathrm{~nm}$ \\
Pulse energy & $110 \mathrm{~mJ} /$ channel \\
Pulse repetition rate & $20.25 \mathrm{~Hz}$ \\
Receiver diameter & $1.0 \mathrm{~m}$ \\
Polarization & Only for the $532 \mathrm{~nm}$ Lidar \\
Footprint/FOV & $100 \mathrm{~m} / 130 \mathrm{urad}$ \\
Vertical Resolution & In the range 30 to $60 \mathrm{~m}$ \\
Horizontal resolution & $5 \mathrm{~km}($ above $8 \mathrm{~km}) / 333 \mathrm{~m}$ (below $8 \mathrm{~km})$ \\
\hline
\end{tabular}

\subsection{Sites under study}

Table 2. CALIOP main parameters

\begin{tabular}{lccc}
\hline Site & $\begin{array}{c}\text { Latitude } \\
\left({ }^{\circ}\right)\end{array}$ & $\begin{array}{c}\text { Longitude } \\
\left({ }^{\circ}\right)\end{array}$ & $\begin{array}{c}\text { Altitude } \\
(\mathrm{m} \mathrm{MSL})\end{array}$ \\
\hline Mauna Kea (13N site) & N 19.8260 & W 155.4747 & 4050 \\
La Palma & N 28.7567 & W 17.8919 & 2250 \\
Kitt Peak & N 31.9583 & W 111.6467 & 2096 \\
Mount Graham & N 32.7013 & W 109.8891 & 3300 \\
Cerro Pachón & S 30.2407 & W 70.7367 & 2750 \\
Cerro Tololo & S 30.1697 & W 70.8068 & 2272 \\
La Silla & S 29.2500 & W 70.7333 & 2400 \\
Las Campanas & S 29.0146 & W 70.6926 & 2380 \\
Paranal & S 24.6272 & W 70.4029 & 2635 \\
Armazones & S 24.5894 & W 70.1919 & 3046 \\
Cerro Honar (Chajnantor) & S 23.0833 & W 67.7667 & 5400 \\
\hline
\end{tabular}

CALIOP Level 2 data for the period 2006 to early 2017, were obtained using the Data Ordering Tool available through the Langley Research Center/NASA web site. The ordering tool allows one to specify the coordinates of a user-defined bounding box around the geographical coordinates of the sites of interest. Since the final orbital 
coverage pattern results in the orbits circling the planet with a separation of about 1.545 degrees, the user-defined bounding box was set of at least $0.5^{\circ} \times 0.5^{\circ}$ and up to $1^{\circ} \times 1^{\circ}$, this to ensure finding a good number of CALIOP observations near the sites of interest, listed in Table 2. Therefore, the bounding box looked for data within about $60 \mathrm{~km}$ to $115 \mathrm{~km}$ around each site of interest.

The CALIOP $532 \mathrm{~nm}$ lidar transmits and detects horizontal and perpendicular polarizations. Hence, two important parameters can be computed, one being the layer-integrated depolarization ratio and another the total layer-integrated attenuated backscatter (see Eq. 1 and Eq. 2 in $^{15}$ ). Also, the lidar is able to determine the cloud base altitude. Together, these parameters can be used for the identification of cloud type, ${ }^{15,16}$ and in particular cirrus clouds. ${ }^{17}$ For instance, randomly oriented crystals in ice clouds can introduce significant depolarization of the signal. ${ }^{18}$ Procedures proposed for the automatic detection of cirrus clouds from lidar data have shown that thin cirrus clouds do not exceed an optical depth of 0.3 nepers. ${ }^{19}$

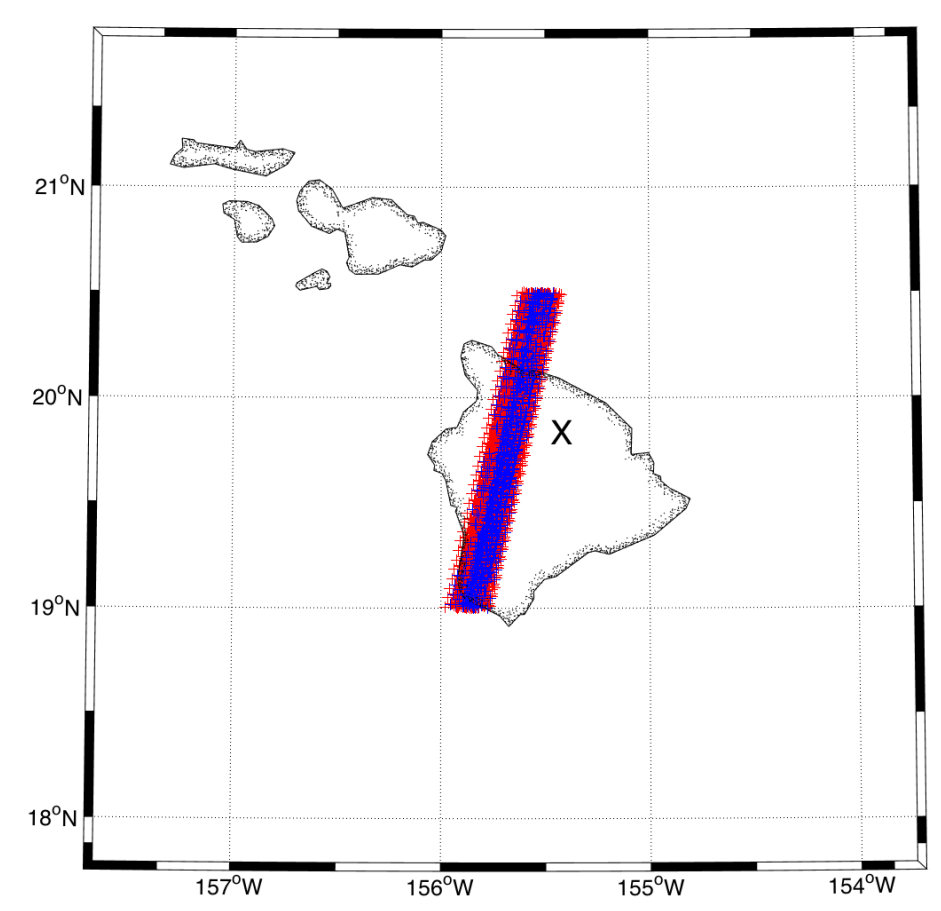

Figure 2. Map of the Big Island of Hawaii. CALIOP nighttime observations in the period 2006-2017 (red crosses), observations in which a thin cirrus cloud (optical depth less than 0.3 nepers) was detected (blue crosses). The $\mathrm{X}$ indicates the location of the observatory site.

Consequently, for this study, we filtered the databases for each site, selecting only the measurements where a single-layer cloud was detected at a geographical altitude higher than the site altitude and for which the optical depth is at most 0.3 nepers. Figure 2 presents a map of the big island of Hawaii that shows the nadir view measurements of the CALIOP $532 \mathrm{~nm}$ lidar in the period 2006 to early 2017 (red crosses), as well as those measurements where a cloud was detected above the altitude of the Maunakea site (blue crosses) and with a maximum cloud optical depth of 0.3 nepers (this corresponds to an extinction of 0.276 magnitudes per airmass).

Similar plots are shown in Figure 8, for the data coverage obtained for all of the other sites of interest in this study.

One important assumption in this study is that the clear-sky/thin-cirrus-cloud conditions determined from the CALIOP data available from the locations nearby to the observatory sites is, in a statistical sense, representative 
of the conditions at the sites. Later on, in the analysis section of this manuscript, it will be shown that the overall statistics agree quite well with previous studies using different datasets. In addition to those results, this work provides a year-to-year, and monthly characterization of thin-cirrus cloud thickness, altitude, and optical depth and also aerosol optical depth. Such data are useful for studies that include propagation of laser light through the atmosphere, laser fratricide effects and atmospheric extinction in various astronomical bands.

\section{STATISTICAL RESULTS}

\subsection{Multi-year statistics of cloud physical properties}

The parameters of interest are:

- Clear-sky fraction.

- Clear-sky + thin-cirrus fraction.

- Thin cirrus cloud mean-base altitude and its standard deviation, as well as cloud thickness.

- Thin cirrus clouds optical depth statistical quartiles.

- Upper-atmosphere aerosols optical depth statistical quartiles.

The dataset was analyzed such as to determine the statistics for the full period of data available, as well as the annual and monthly variability.

Table 3 summarizes the full-period statistics for each of the sites listed in Table 2 .

If the statistics of clear sky plus thin-cirrus clouds fraction can be interpreted as what in other studies is called the usable-time fraction or spectroscopy sky quality fraction, then the overall statistics shown in Table 3, from the analysis of the CALIP database, matches very well the results from previous studies. The work of Andre Erasmus and van Staden, ${ }^{20}$ from the analysis of GOES- 8 satellite $10.7 \mathrm{um}$ channel observations, found a usable fraction of $78.2 \%$ for the Cerro Tololo site, $83.1 \%$ for La Silla Las Campanas area, and $90.5 \%$ for the Paranal/Armazones area. For these sites, the results in this study are in agreement, within 1.5\%, with the Erasmus \& van Staden results. In the particular case of La Silla observatory, long-term monitoring of photometric sky quality (1984 through 2013$)^{\dagger}$, shows the mean photometric fraction to be $63 \%$ with a standard deviation of $20 \%$ (see footnote 3 ), this result is compatible with the $75 \%$ clear sky fraction found in this study. The main difference is for the Mauna Kea site, while in this study the data shows an overall $81 \%$ of usable time, the Erasmus study indicates $71.4 \%$.

For the La Palma site, from the analysis of observing log-books of the Telescopio Nazionale Galileo (TNG), in the period 2000 to 2008 , a percentage of clear nights of $62.6 \%$ was found. ${ }^{21}$ This result compares well with the $64 \%$ found in the present study. However, the same study (della Valle et al., 2010) found a $71.9 \%$ clear sky fraction for the site of La Palma from the analysis of GOES-12 satellite observations with the 10.7 um channel. For the Kitt Peak site, and from the nighttime observing report compiled by D.L. Crawford ${ }^{\ddagger}$, the overall annual mean usable sky fraction is estimated to be $70 \%$, this agrees quite well with the $72 \%$ of usable time found in this study. The Mount Graham and Kitt Peak are within the same climatological region in the USA southwest, but when analyzing the CALIOP sensor data separately, using only the data closer to each site (see CALIOP data coverage in Figure 8, top-right panel), the results show Kitt Peak to have about $5 \%$ larger clear-sky and usable-sky fractions than Mount Graham. However, this $5 \%$ variability is in the order of magnitude of the overall year-to-year variability in the data.

In summary, the overall statistics of clear fraction and usable time at astronomical sites, determined from CALIPSO data, generally agree very well with the results known from the observatory statistics or previous studies.

\footnotetext{
${ }^{\dagger}$ http://www.eso.org/gen-fac/pubs/astclim/paranal/clouds/cloudys-1984-2013.jpg

${ }^{\ddagger}$ https://www.noao.edu/kpno/usrhnbk/user-App.html
} 
Table 3. Overall, 2006-2017, statistical results of cloud thickness, thin cirrus and aerosol optical depths, clear sky fraction and clear+thin-cirrus sky fraction for selected astronomical sites. For cloud base, cloud top and cloud thickness, the statistics are mean value \pm one standard deviation. Q1, Q2 and Q3 are the $25 \%, 50 \%$ and $75 \%$ quartiles.

\begin{tabular}{lrrrrrrr}
\hline Parameter & MK & ORM & KP/MG & CP/CT & LS/LC & Par/Arm & Honar \\
\hline No. of data points & 5452 & 5687 & 29801 & 4846 & 3934 & 7387 & 5492 \\
Q1 cloud thick. (km) & 0.778 & 0.838 & 0.719 & 0.599 & 0.599 & 0.659 & 0.599 \\
Q2 cloud thick. (km) & 1.078 & 1.138 & 1.018 & 0.898 & 0.958 & 0.839 & 0.898 \\
Q3 cloud thick. $(\mathrm{km})$ & 1.557 & 1.617 & 1.460 & 1.377 & 1.392 & 1.235 & 1.317 \\
Cloud base alt. $(\mathrm{km})$ & $11.7 \pm 2.7$ & $9.3 \pm 2.0$ & $9.6 \pm 2.8$ & $9.7 \pm 1.9$ & $9.5 \pm 2.0$ & $11.1 \pm 2.6$ & $11.7 \pm 2.5$ \\
Cloud top alt. $(\mathrm{km})$ & $12.9 \pm 2.7$ & $10.7 \pm 2.1$ & $10.8 \pm 3.0$ & $10.7 \pm 2.0$ & $10.7 \pm 2.1$ & $12.1 \pm 2.7$ & $12.8 \pm 2.5$ \\
Cloud thick. $(\mathrm{km})$ & $1.26 \pm 0.71$ & $1.33 \pm 0.75$ & $1.19 \pm 0.69$ & $1.04 \pm 0.54$ & $1.15 \pm 0.75$ & $1.03 \pm 0.60$ & $1.07 \pm 0.60$ \\
Q1 thin cirrus $\tau$ & 0.012 & 0.025 & 0.019 & 0.031 & 0.030 & 0.018 & 0.014 \\
Q2 thin cirrus $\tau$ & 0.049 & 0.074 & 0.070 & 0.083 & 0.090 & 0.051 & 0.048 \\
Q3 thin cirrus $\tau$ & 0.126 & 0.154 & 0.151 & 0.179 & 0.190 & 0.124 & 0.117 \\
Q1 aerosol $\tau$ & 0.023 & 0.029 & 0.016 & 0.011 & 0.011 & 0.013 & 0.009 \\
Q2 aerosol $\tau$ & 0.060 & 0.063 & 0.041 & 0.030 & 0.027 & 0.025 & 0.023 \\
Q3 aerosol $\tau$ & 0.131 & 0.140 & 0.095 & 0.065 & 0.059 & 0.046 & 0.047 \\
Clear-sky sections & 3603 & 3662 & 18196 & 3316 & 2946 & 5935 & 4246 \\
Thin cirrus sections & 819 & 535 & 3348 & 414 & 274 & 676 & 560 \\
Clear-sky fract. & $66 \%$ & $64 \%$ & $61 \%$ & $68 \%$ & $75 \%$ & $80 \%$ & $77 \%$ \\
Thin-cirrus fract. & $15 \%$ & $10 \%$ & $11 \%$ & $9 \%$ & $7 \%$ & $9 \%$ & $11 \%$ \\
Usable fract. & $81 \%$ & $74 \%$ & $72 \%$ & $77 \%$ & $82 \%$ & $89 \%$ & $88 \%$ \\
\hline
\end{tabular}

\subsection{Monthly statistics of clear sky and usable fractions}

For each of the sites under study, the CALIOP data gathered in the period 2006-2017 were grouped by month of the year. This allows one to estimate the monthly statistics for cloud coverage as well as cloud properties. However, one should keep in mind, that the data in each month comes from orbits of the CALIPSO satellite over the given sites in multiple years, and this averages out the annual variability. The results for monthly clear sky fraction and monthly usable fraction (including those days with thin cirrus clouds) are shown in Figures 3 and 4 , respectively. Figure 5 shows the mean clear-sky and usable night fractions for each year.

Qualitatively, the clear fraction and usable fraction seem to agree quite well with direct experience. For instance, the increasing trend of clear/usable fractions in the northward direction for the Chile sites (moving away from the possible adverse effects of mid-latitude weather fronts). The relatively-lower fraction of clear/usable time at the sub-tropical zone (Cerro Pachn and Cerro Tololo) is likely because those sites are more prone to be affected by weather fronts moving north from the mid-latitudes. While, the sites such as Paranal and Armazones lie in the latitude of the descending (subsiding) branch of the Hadley cell. This subsiding dry air creates a high-pressure center that warms the mid-levels of the atmosphere and lower troposphere. This effect, together with the cold Humboldt current along the eastern Pacific basin, creates the conditions that ultimately allow a higher fraction of clear sky. ${ }^{22}$

Interestingly enough, the CALIOP lidar results show that the median aerosol optical depth is about a factor of two larger for the northern-hemisphere sites than for the southern-hemisphere sites. A likely explanation for this is the higher fraction of continental masses, higher industrialization, but also of regions known of generating and distributing mineral dust from the surface into the higher levels of the troposphere, such as the Sahara. ${ }^{23}$ The results in this study show a 50\% percentile aerosol optical depth of 0.063 for the site of Roque de Los Muchachos in the period 2006-2017 (mean value 0.060, excluding the high-aerosol events associated with the advection of Saharan dust). Lidar observations done in situ for a few nights in 2007 and 2008, in the context of the ELT site testing effort, show an average value of 0.0405 at $532 \mathrm{~nm}$ (in standard conditions) and as high as 0.233 during Saharan dust advection events. ${ }^{24}$ Using the aerosol optical thickness results shown in Figure 7 of, ${ }^{24}$ 


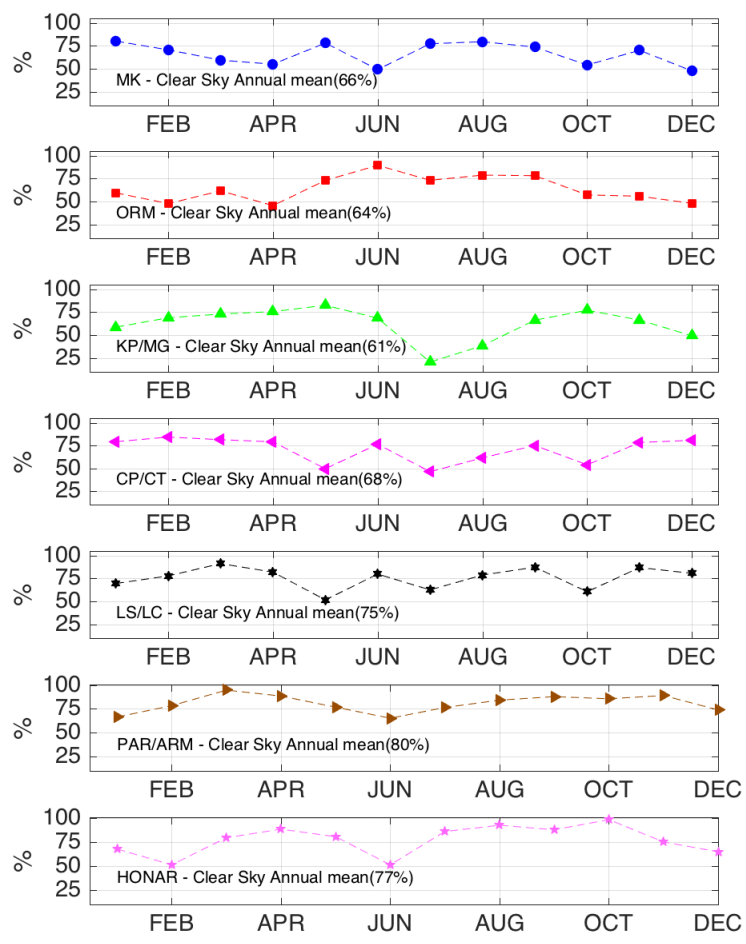

Figure 3. Monthly Clear Sky Fraction. The sites are: Mauna Kea (MK), La Palma (ORM), Kitt Peak/Mount Graham KP/MG, Cerro Pachn/Cerro Tololo (CP/CT), La Silla/Las Campanas (LS/LC), Paranal/Armazones (Par/Arm), Cerro Honar (HONAR).

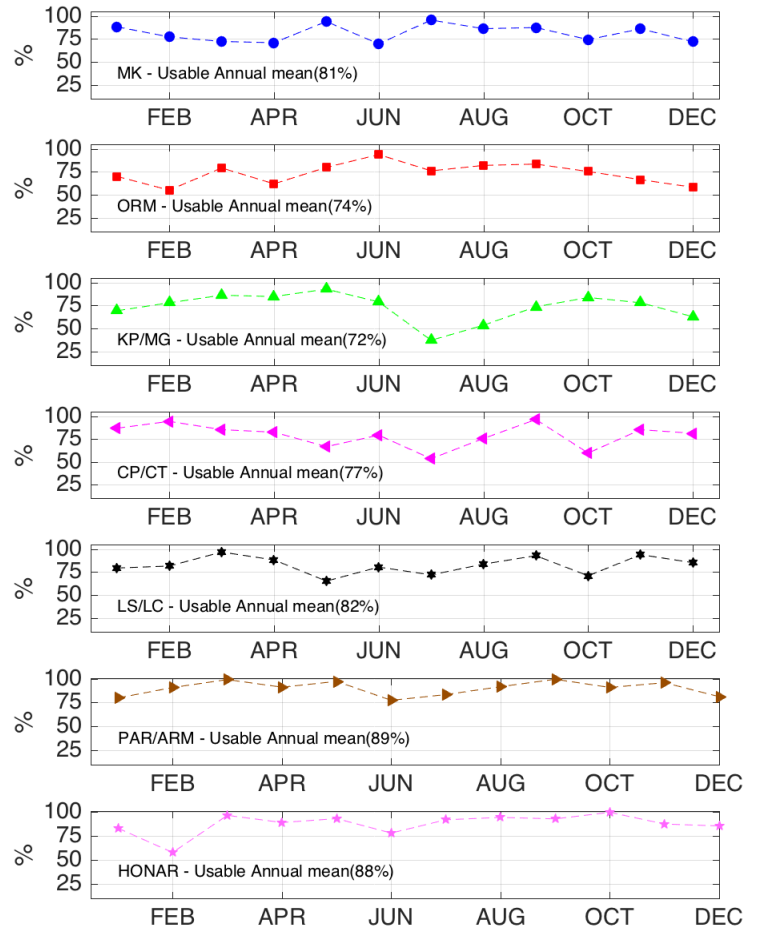

Figure 4. Monthly Usable Fraction (i.e. includes the effect of thin-cirrus clouds with optical depth up to 0.3 nepers). The site labels are as in Figure 3.

and including only those data points that have optical thickness less than 0.15 nepers (to remove the potential bias of Saharan dust events), the variability of the remaining values is 0.038 nepers. Consequently, the statistical results obtained in this study for aerosol optical depth are compatible with the limited in-situ measurements of aerosol extinction.

On the other hand, the mean cloud base and cloud top altitudes, are higher at sites located by the tropical region when compare to the sites of higher latitudes. This is consistent with the expanded atmosphere due to higher energy-content in the atmospheric column in the tropical latitudes. Also, the monthly distributions of clear/usable sky fraction (See Figure 3), show the clear effect of the North American monsoon (summer months) at the Kitt Peak and Mount Graham sites, when it is mostly cloudy, as well as the seasonal (winter/summer) signal for all sites.

\subsection{Cirrus cloud optical depth variability}

The CALIOP Level 2 data consists of 5-km sections along the path of the satellite. The CALIOP $532 \mathrm{~nm}$ lidar footprint is only $90 \mathrm{~m}$ in diameter, so in order to achieve a sufficiently-high signal to noise ratio for the detection of cirrus clouds, the data from this sensor is averaged in sections of $5 \mathrm{~km}$ in length (equivalent to about 55 individual footprint-size sections). Of interest in this study is the variability of cirrus clouds optical depth on short time scales, or short spatial lengths, for instance comparable to the size of a laser beam that propagates through the atmosphere (of order 20 to $50 \mathrm{~cm}$ laser beam diameter). In the case of the TMT project, the laser 

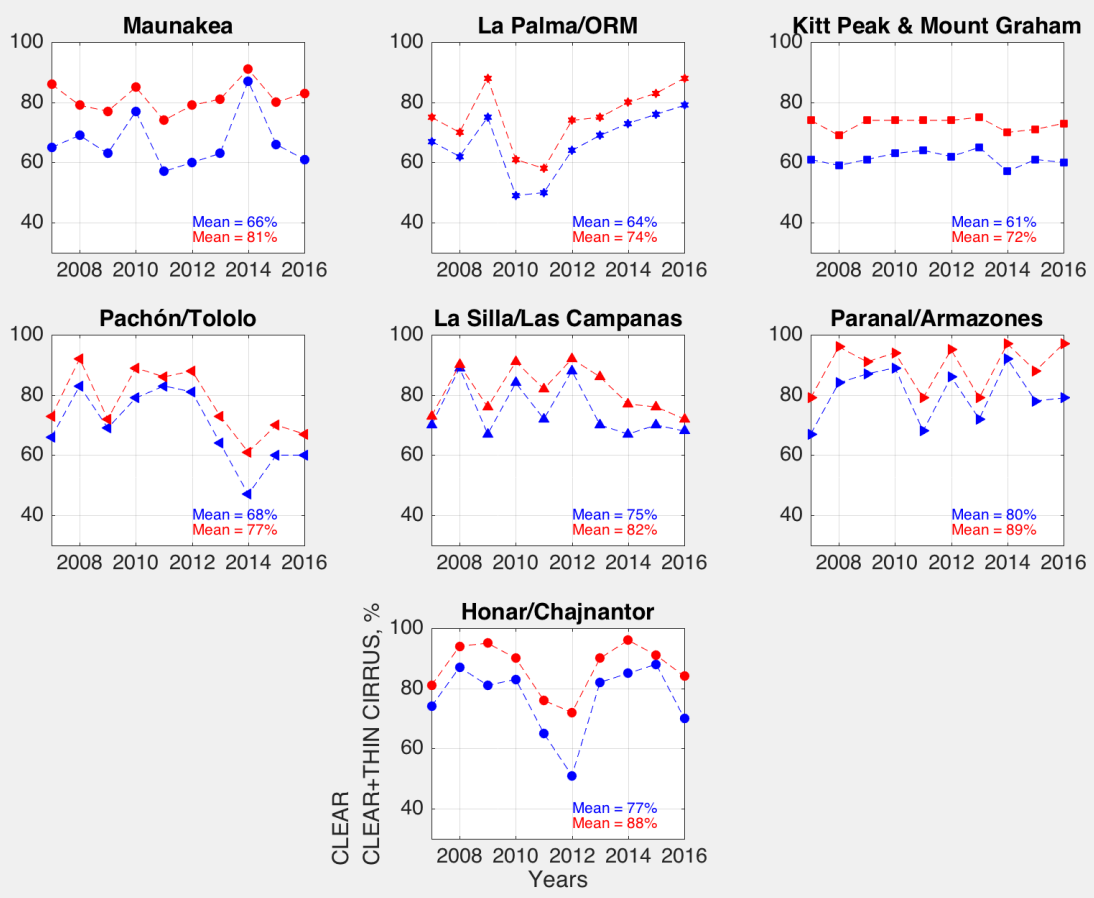

Figure 5. Annual statistics of clear-sky (blue) and usable-nights (red) fractions for the sites under study. The mean overall annual statistics for clear sky(blue) and clear+thin-cirrus(red) fractions are also shown.

has a Gaussian intensity profile, having diameter, to the $1 / e^{2}$ intensity level, of $24 \mathrm{~cm}$, i.e. very short when compare to the $5 \mathrm{~km}$ sections of CALIOP sensor data. If a cirrus cloud is located at 8000 meters above the telescope site, then for a laser propagating at 45 degrees zenith angle, the $24 \mathrm{~cm}$ diameter subtends and angle of about 0.5 arcsec. If the cloud layer drifts with a mean wind of $20 \mathrm{~m} / \mathrm{s}$, the cirrus cloud section will cross the laser beam diameter in only $12 \mathrm{~ms}$.

The variability of cirrus clouds can be studied by time-resolved stellar photometry, while observing through thin cirrus clouds. To this end, a series of exposures was obtained using a Canon 60D digital single-lens reflex (DSLR) photographic camera equipped with a 250-mm focal length telephoto lens, for two reference stars. Exposures were taken every 2 seconds, with an exposure time of $200 \mathrm{~ms}$. The camera was slightly defocused in order to avoid saturation, as well as to spread the light of the star over several pixels and thus improve the photometry. Aperture photometry, using the green channel, was then used to estimate the stellar fluxes. Figure 6 shows the histograms for the variability in the photometry from observations of Arcturus and Vega. The time series shown in the upper panel of Figure 6 is that from observations of Arcturus. The first part of the observations show a larger variability. This was found to be due to stray light so a baffle was added to protect the lens. The histograms indicate variability at the 1 -sigma level of $0.1 \mathrm{mag} /$ airmass to $0.3 \mathrm{mag} / \mathrm{airmass}(10 \%$ to $30 \%$ ), respectively. The $10 \%$ to $30 \%$ level fluctuations are larger than the stellar brightness fluctuations expected only from scintillation, which, for the 58-mm aperture of the lens used in these observations is expected to be of about $4 \%$ to $5 \%$ [25, Eq. 2].

\section{ATTENUATION AND VARIABILITY IN THE ATMOSPHERIC PATH}

The TMT first-light baseline plan consists on using six lasers that create the asterisms necessary to support the adaptive optics correction system. The plan includes the use of TOPTICA/MPB lasers consisting of continuouswave laser light of $22 \mathrm{~W}$ power at $589 \mathrm{~nm}$ wavelength, for the excitation of the $\mathrm{D}_{2 \mathrm{a}}$ line of sodium atoms in the 
mesosphere. It allows up to $10 \%$ of the laser power to be used for re-pumping ${ }^{\S}$ of the $\mathrm{D}_{2 \mathrm{~b}}$ line. ${ }^{26,27}$ As the laser light propagates upward, it will experience losses due to scattering of light out of the beam due to small optics aberrations in the beam transfer and laser launch telescope optical surfaces, as well as due to scattering by air molecules, aerosols and cirrus cloud particles along the atmospheric channel in the propagation of the laser beam from the telescope to the mesosphere. The TMT has a requirement for the beam transfer optics throughput of $75 \%$ (i.e. allow losses not to exceed $25 \%$ due to scattering of light out of the main beam arising from aberrations in the optical surfaces and absorption in optical surface coatings).

For the atmospheric channel, the scattering can be modeled by means of molecular Rayleigh scattering theory. The molecular angular volume scattering coefficient is the fraction of photons scattered out of laser light beam per steradian per meter of laser beam path. It is proportional to the molecular scattering cross-section, the number density of molecules, and the Rayleigh scattering phase function. A set of equations describing this process is included in the work of Wang et al. ${ }^{12}$ A similar formalism has been used in this study to estimate the optical depth of the atmosphere, at $589 \mathrm{~nm}$ wavelength, arising from Rayleigh molecular scattering for each site listed in Table 2. The vertical profile of the molecular number density, for each site, was obtained from the US standard atmosphere [28, Table A.8, p. 1293]. Tables 4 summarizes the contributions to the total optical depth $(\tau)$ from molecular, aerosol and cirrus scattering, and the overall atmospheric transmission $\left(T_{\text {atm }}=e^{-\tau}\right)$, for $589 \mathrm{~nm}$ and $532 \mathrm{~nm}$ wavelength respectively.

Table 4. Optical depth at the various sites in this study, including contribution from aerosols, cirrus, Rayleigh scattering, and the Chappuis Ozone band, at wavelenghts of 589 and $532 \mathrm{~nm}$. The total optical depths and atmospheric transmission are computed using the median values for aerosol and cirrus cloud topical depths learned from the analysis of CALIOP sensor lidar data. Aerosol and cirrus optical depths are given for a wavelength of $532 \mathrm{~nm}$, and are the same for $589 \mathrm{~nm}$.

\begin{tabular}{lcccccccccccc}
\hline Site & \multicolumn{2}{c}{$\tau_{\text {aerosol }}$} & \multicolumn{2}{c}{$\tau_{\text {cirrus }}$} & \multicolumn{2}{c}{$\tau_{\text {Rayleigh }}$} & \multicolumn{2}{c}{$\tau_{\text {ozone }}$} & \multicolumn{3}{c}{$\tau_{\text {total }}$} & \multicolumn{2}{c}{ Atm. trans. } \\
& $25 \%$ & $50 \%$ & $25 \%$ & $50 \%$ & 589 & 532 & 589 & 532 & 589 & 532 & 589 & 532 \\
\hline MK & 0.023 & 0.060 & 0.012 & 0.049 & 0.045 & 0.067 & 0.031 & 0.018 & 0.185 & 0.194 & $83 \%$ & $82 \%$ \\
ORM & 0.029 & 0.063 & 0.025 & 0.074 & 0.056 & 0.085 & 0.031 & 0.018 & 0.224 & 0.240 & $80 \%$ & $79 \%$ \\
KP & 0.016 & 0.041 & 0.019 & 0.070 & 0.057 & 0.087 & 0.031 & 0.018 & 0.199 & 0.216 & $82 \%$ & $81 \%$ \\
MG & 0.016 & 0.041 & 0.019 & 0.070 & 0.049 & 0.074 & 0.031 & 0.018 & 0.191 & 0.203 & $83 \%$ & $82 \%$ \\
CP & 0.011 & 0.030 & 0.031 & 0.083 & 0.053 & 0.080 & 0.031 & 0.018 & 0.197 & 0.211 & $82 \%$ & $81 \%$ \\
CT & 0.011 & 0.030 & 0.031 & 0.083 & 0.056 & 0.085 & 0.031 & 0.018 & 0.200 & 0.216 & $82 \%$ & $81 \%$ \\
LS & 0.011 & 0.027 & 0.030 & 0.090 & 0.055 & 0.083 & 0.031 & 0.018 & 0.203 & 0.218 & $82 \%$ & $80 \%$ \\
LC & 0.011 & 0.027 & 0.030 & 0.090 & 0.055 & 0.084 & 0.031 & 0.018 & 0.203 & 0.219 & $82 \%$ & $80 \%$ \\
Par & 0.013 & 0.025 & 0.018 & 0.051 & 0.053 & 0.081 & 0.031 & 0.018 & 0.160 & 0.175 & $85 \%$ & $84 \%$ \\
Arm & 0.013 & 0.025 & 0.018 & 0.051 & 0.051 & 0.077 & 0.031 & 0.018 & 0.158 & 0.171 & $85 \%$ & $84 \%$ \\
Honar & 0.009 & 0.023 & 0.014 & 0.048 & 0.037 & 0.056 & 0.031 & 0.018 & 0.139 & 0.145 & $87 \%$ & $87 \%$ \\
\hline
\end{tabular}

The aerosol and cirrus optical depths correspond to the $25 \%$ and $50 \%$ percentiles obtained from the CALIOP $532 \mathrm{~nm}$ wavelength lidar data, and reported in Table 3. For Mie scattering, aerosols and cirrus clouds, the optical depths for monochromatic light at $589 \mathrm{~nm}$ are about the same as those at $532 \mathrm{~nm}$. This, because their scattering efficiency are about the same, $Q_{\text {sca }}(532 \mathrm{~nm}) \simeq Q_{\text {sca }}(589 \mathrm{~nm})$, where $Q_{\text {sca }}$ is the scattering efficiency coefficient. However, for Rayleigh scattering the optical depths will scale as the inverse of wavelength to the 4th power, $\tau^{R}(589 \mathrm{~nm})=\tau^{R}(532 \mathrm{~nm})(532 / 589)^{4}$.

For completeness, the optical depth due to absorption by the Chappuis band, arising from ozone molecules in the mesosphere, was included. The ozone optical depth is extracted for the $532 \mathrm{~nm}$ and $589 \mathrm{~nm}$ wavelengths, in units of mag/airmass, from Figure $15 \mathrm{c}$ in the work of Buton et al. ${ }^{29}$ and converted into optical depth using $\tau=0.9210 \times$ magnitude.

\footnotetext{
${ }^{\S}$ TOPTICA/MPB defines the re-pumping power fraction $(q)$ as the fraction of the power in the Na $D_{2 b}$ line over the power that goes to the $\mathrm{Na} \mathrm{D}_{2 \mathrm{a}}$ line $\left(q=P_{\mathrm{D} 2 \mathrm{~b}} / P_{\mathrm{D} 2 \mathrm{a}}\right)$.
} 
Simulations of the propagation of the 589-nm wavelength laser beam through the attenuating atmosphere where performed using the LGSBloch package ${ }^{\Uparrow}$, implemented in Mathematica@, as an extension to the Atomic Density Package (a collaboration of researchers Simon Rochester, at Rochester Scientific LLC, and Ron Holzlhner at the European Southern Observatory).

Table 5 shows the parameters used in the simulations for two sites, Mauna Kea and the Observatorio de Roque de Los Muchachos.

Table 5. Parameters of the site and TMT laser used to estimate the LGS photon flux at two sites.

\begin{tabular}{|c|c|c|}
\hline Parameter & Mauna Kea 13N & ORM \\
\hline Zenith Angle & $0^{\circ}$ & $0^{\circ}$ \\
\hline Laser Power & $20 \mathrm{~W}$ & $20 \mathrm{~W}$ \\
\hline Laser polarization & circular & circular \\
\hline Laser spectral bandwidth & $1.34 \mathrm{MHz}^{30}$ & $1.34 \mathrm{MHz}^{30}$ \\
\hline $\mathrm{D}_{2 \mathrm{~b}}$ re-pumping $(q)$ & $10 \%$ & $10 \%$ \\
\hline $\mathrm{Na}$ column density & $4 \times 10^{13}$ atoms $/ \mathrm{m}^{2}$ & $4 \times 10^{13}$ atoms $/ \mathrm{m}^{2}$ \\
\hline LGS spot size (FWHM) & 0.6 arcsecs & $0.6 \operatorname{arcsecs}$ \\
\hline Geomagnetic field $|B|$ & $3.33 \times 10^{-5} \mathrm{~T}$ & $3.70 \times 10^{-5} \mathrm{~T}$ \\
\hline Magnetic zenith & $36.41^{\circ}$ & $38.01^{\circ}$ \\
\hline Magnetic azimuth & $189.57^{\circ}$ & $185.98^{\circ}$ \\
\hline Site altitude & $4050 \mathrm{~m}$ & $2250 \mathrm{~m}$ \\
\hline Atmospheric optical depth & 0.185 nepers & 0.224 nepers \\
\hline Atmospheric transmission & $83 \%$ & $80 \%$ \\
\hline Photon flux return at site & $16.5 \times 10^{6}$ photons $/ \mathrm{m}^{2} / \mathrm{s}$ & $14.7 \times 10^{6}$ photons $/ \mathrm{m}^{2} / \mathrm{s}$ \\
\hline TMT detection throughput & 0.3477 & 0.3477 \\
\hline NFIRAOS frame rate & $800 \mathrm{~Hz}$ & $800 \mathrm{~Hz}$ \\
\hline NFIRAOS subaperture area & $0.52 \mathrm{~m}^{2}$ & $0.52 \mathrm{~m}^{2}$ \\
\hline Detected photoevents & 1793 photons/sub-aperture/frame & 1597 photons/sub-aperture/frame \\
\hline
\end{tabular}

Fluctuations of $\pm 30 \%$ in the cirrus optical depth, in time scales of a few seconds, will induce fluctuations of order $5 \%$ in the overall photo-detected-events. This $5 \%$ fluctuation will be in addition to other fluctuations, such as the natural variability of the sodium atoms column density. ${ }^{31}$ For the particular case of the ORM site, in the extreme case of a dust event characterized by an extinction of $0.2 \mathrm{mag} / \operatorname{arcsecs}(0.184$ nepers) and a cirrus optical depth of 0.154 (75\% percentile, as shown in Table 3), the atmospheric transmission will drop from $80 \%$ to $65 \%$, in which case the photo-detected events will decrease to 1087 photons/sub-aperture/frame. This value is slightly above the TMT requirement of 900 photons/sub-aperture/frame needed for the successful determination of the LGS centroids in each sub-aperture in order to achieve the goal of $187 \mathrm{~nm}$ maximum wavefront error along the boresight direction.

\section{CIRRUS VARIABILITY AND LASER FRATRICIDE}

Laser fratricide affects multi-laser AO systems, particularly when the laser beams are launched from behind the telescope secondary mirror. The term fratricide is used here in the sense that back-scattered photons, in the propagation of a given laser (in the multiple lasers configuration) are detected by the wavefront sensors observing the LGS created by other lasers in the system. The backscattering of photons arises from the interaction of the laser beam light with air molecules, as well as atmospheric aerosols (dust particles, or aerosols results of photochemical aggregation) and ice particles in cirrus clouds. This effect has been first documented and studied by the Gemini MCAO System team. ${ }^{10}$ As the TMT laser system is also a center-launched multi-laser system, it is prone to this effect. The TMT adaptive optics team has studied and planned strategies for calibrating out the fratricide pattern. ${ }^{12}$ They also estimated the effect of variability in the cirrus optical depth.

\footnotetext{
『 http://budker.berkeley.edu/ADM/
} 


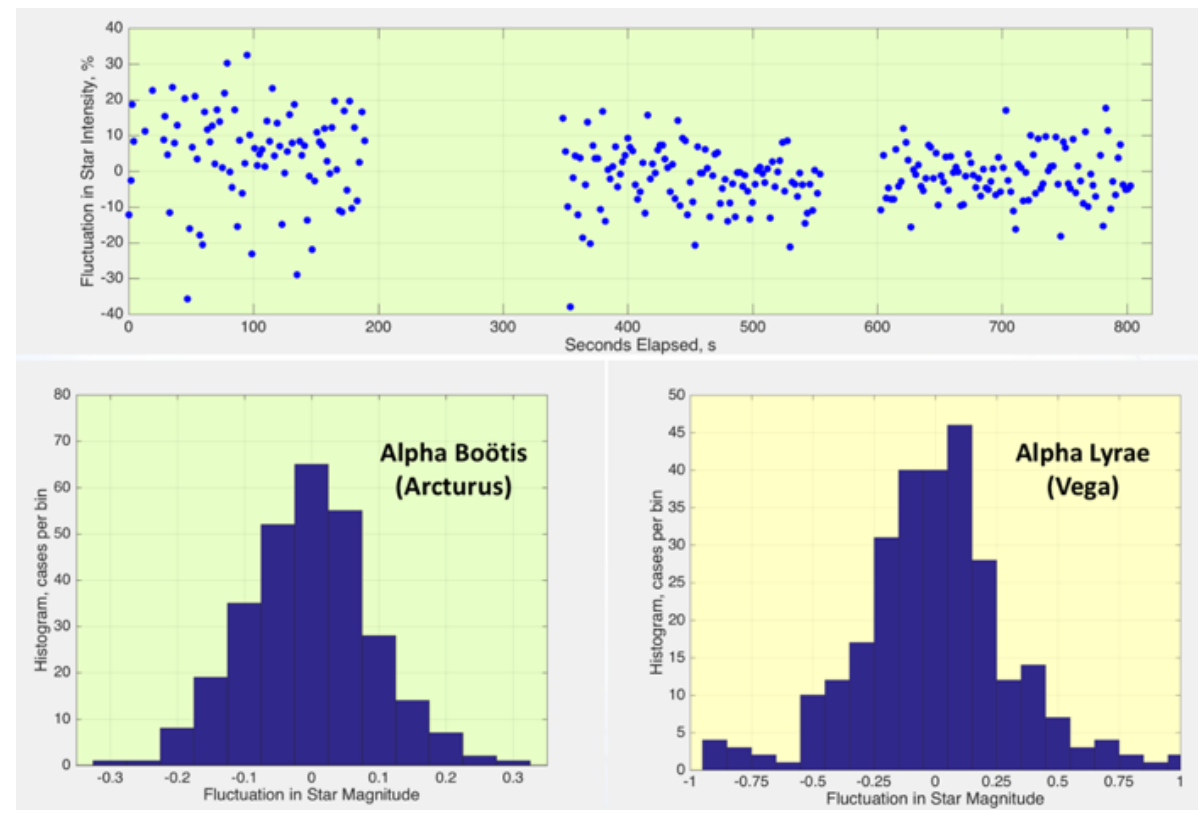

Figure 6. Variability in the photometry of a couple reference stars, attributed to fluctuations in atmospheric extinction with sources in a thin cirrus cloud. 1-sigma level photometry variability was about 10\% (Arcturus), and 30\% (Vega). The upper panel shows the time series in the photometry of Arcturus.

Figure 7 shows the modeled backscattering pattern detected by the WFS-0 CCD, that associated with the observation of the central LGS in the TMT MCAO asterism. Sub-apertures (SA) detecting backscattered photons from a given altitude above ground level $(h)$, will depend on the orientation of the LGS asterism and their angular radial aperture $(\theta)$. The first light TMT adaptive optics system uses a 6-LGS asterism, with one of the LGS in the boresight direction and the five other LGS equidistance distributed at 72 degrees azimuthal separation and angular radial aperture $(\theta)$ of 35 arcsec. Photons backscattered from an altitude of about $10 \mathrm{~km}$, comparable with the location of cirrus clouds, will mainly affect the most inner wavefront sensor apertures. The number of photons detected, decreases radially out in the affected WFS sub-apertures since the photons originate at higher levels into the atmosphere (i.e. lower molecular density).

The temporal variability in the intensity of the laser fratricide pattern in the WFS apertures is a function of a) temporal variability in the laser power at the source, and b) temporal changes in the extinction induced by the cirrus cloud. In the case of the GeMS, it was found that the variability in the intensity of the fratricide pattern likely had a strong component arising from the laser power variability. ${ }^{11}$ This should soon be reduced as the GeMS team plans to add a more stable laser supplied by TOPTICA/MPB ${ }^{32}$ having power stability better than 1\%. TMT also plans on using similar type of laser. Consequently, the main contribution to the fluctuation in the laser fratricide pattern will likely arise from cirrus cloud extinction, which as reported in this study can be of order $30 \%$ in time scales of a few seconds (see Figure 6).

\section{CONCLUSIONS}

The $532 \mathrm{~nm}$ LIDAR included in the CALIOP sensor onboard of the CALIPSO satellite was used for the identification of cirrus clouds with optical depth up to 0.3 nepers (thin cirrus) for the sites of existing astronomical observatories. The data helped estimate the clear fraction and usable fraction of nights through the year at those sites. The results from this analysis are consistent with results from previous studies. In addition to those studies the data used in this report has been used to obtain the monthly variability of clear and usable nights in a year, as well as relevant statistics for the cirrus clouds and aerosols optical depths for those sites at observatory locations. The information, given at $532 \mathrm{~nm}$ and $589 \mathrm{~nm}$ wavelength can be used for modeling of the propagation of laser beams through the atmosphere supporting adaptive optics systems. Finally, the effect 


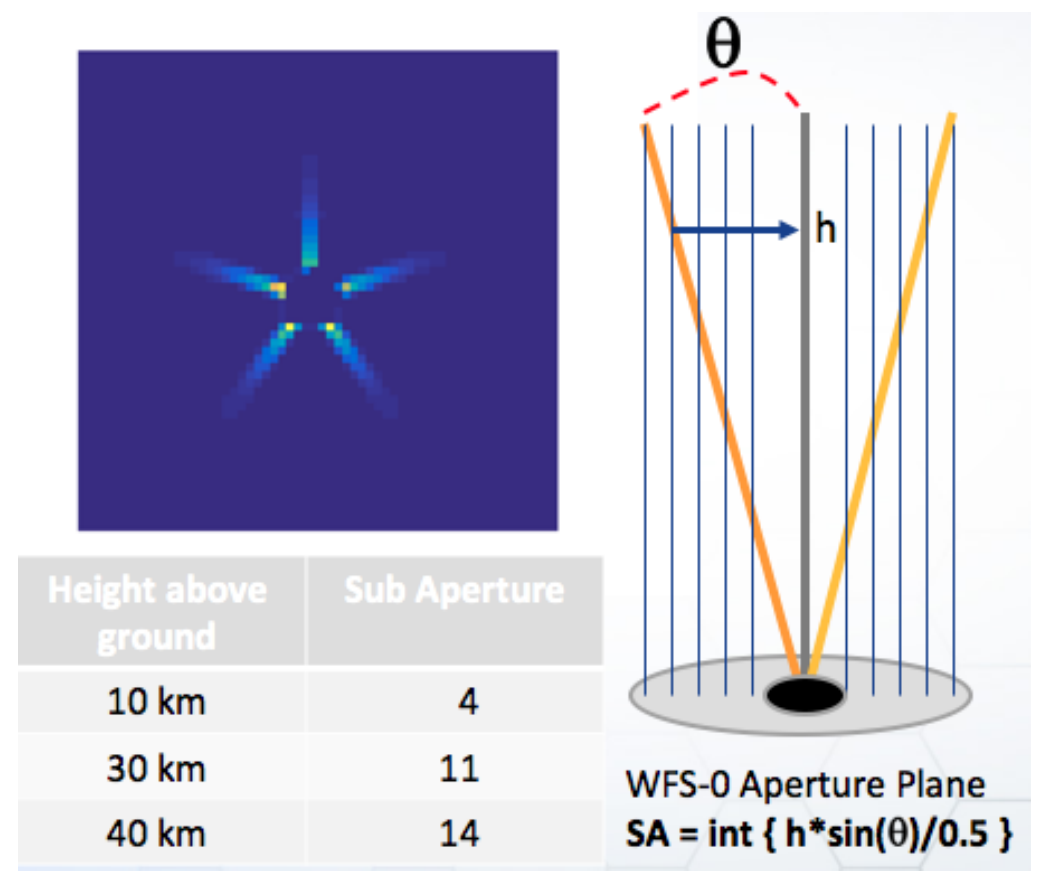

Figure 7. TMT WFS0 Laser Fratricide Pattern. Pattern obtained with the help of a Matlab@ routine (backscatter.m) written and provided by Lianqi Wang (TMT AO Team). The TMT WFS sensor includes $60 \times 60$ sub-apertures of size $0.5 \mathrm{~m} \times 0.5 \mathrm{~m}$.

of temporal variability in the cirrus clouds optical depth on the intensity of the laser guide stars, as well as in the variability of the laser fratricide pattern was considered.

The main results are the following:

- For the astronomy sites in northern Chile, there is a clear northward trend of increasing clear-sky/usablenights fraction. This can be attributed to the decreasing effect of mid-latitude weather fronts as the sites are located further north into the Atacama Desert. Cerro Pachón and Cerro Tololo sites have the lowest clear-sky/usable-nights fraction, although still quite good at $68 \% / 77 \%$, respectively. These fractions are higher than those found for the Mount Graham/Kitt Peak observatory sites in the northern hemisphere.

- The cirrus cloud altitude is higher for sites located closer to the equator, and lower for sites in the subtropical latitudes. This is consistent with the larger expansion of the atmosphere in the lower latitudes due to the higher mean temperature of the atmospheric column.

- The aerosol column optical depth statistics show there is a higher content of aerosols (such as atmospheric dust) for the northern hemisphere sites, which is of order a factor of two larger than for the southern hemisphere sites. This is perhaps explained by the larger fraction of continental masses, higher degree of industrialization, and geographic locations (such as the northern Sahara and the Mongolian desert) where dryness and atmospheric conditions allow incursion of surface dust into higher levels of the atmosphere.

- Of interest for the TMT project, the fraction of annual usable nights for Mauna Kea and ORM are comparable within 5\%. The results shown in Figures 3 and 4, seem to indicate a slightly more favorable clear-sky and usable-night fractions in winter season for the Mauna Kea site. The annual statistics of clear-sky/usable-nights annual fraction indicate that ORM site has had a larger fraction since about 2012 (see Figure 5). This trend can been explained as due to the persistence of a positive phase of the North Atlantic Oscillation (NAO). A positive phase seems to favor lower precipitation, likely lower cloudiness level at the location of the Canary Islands (TMT Climate Study, internal report prepared by Dr. Edward Graham, Meteorologist and Climatologist). 
- The data clearly shows the adverse effect of the North American monsoon in the fraction of clear-sky/usablenights in summer season at the USA southwest sites. This is why observatories in the region allocate the time for major technical upgrades and overall maintenance of their astronomical facilities in summer time.

- Stellar photometry shows variations of order 30\% (0.1-0.3 mag) for thin-cirrus clouds on timescales of a few seconds. This will affect both the LGS brightness and the intensity and shape of the fratricide pattern for multiple-laser $\mathrm{AO}$ systems.

\section{ACKNOWLEDGEMENTS}

The TMT Project gratefully acknowledges the support of the TMT collaborating institutions. They are the Association of Canadian Universities for Research in Astronomy (ACURA), the California Institute of Technology, the University of California, the National Astronomical Observatory of Japan, the National Astronomical Observatories of China and their consortium partners, and the Department of Science and Technology of India and their supported institutes. This work was supported as well by the Gordon and Betty Moore Foundation, the Canada Foundation for Innovation, the Ontario Ministry of Research and Innovation, the National Research Council of Canada, the Natural Sciences and Engineering Research Council of Canada, the British Columbia Knowledge Development Fund, the Association of Universities for Research in Astronomy (AURA), the U.S. National Science Foundation and the National Institutes of Natural Sciences of Japan. PH acknowledges financial support from the Natural Sciences and Engineering Research Council of Canada. 

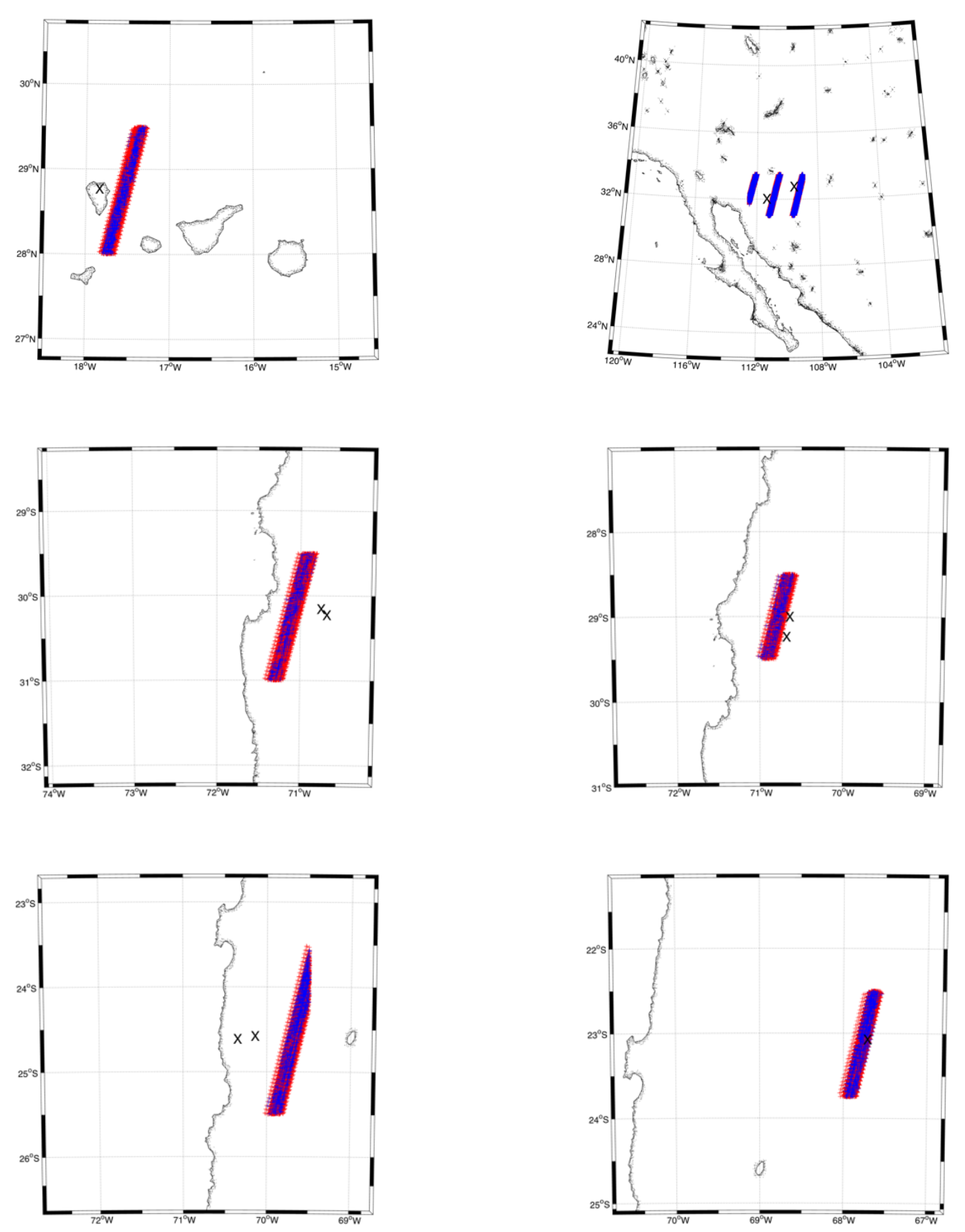

Figure 8. CALIOP nightime data tracks (2006-2017) found in the vicinity of the observatory sites listed in Table 1 . The red crosses indicate all measurements, blue crosses indicates those measurements in which a cirrus cloud with optical depth up to 0.3 nepers is detected by the CALIOP algorithm. The sites are: La Palma (top-left), Kitt Peak/Mount Graham (top-right), Cerro Pachn/Cerro Tololo (center-left), La Silla/Las Campanas (center-right), Cerro Paranal/Cerro Armazones (bottom-left), Cerro Honar (bottom-right). The observatory site locations are indicated with X. 


\section{REFERENCES}

[1] Beniston, M., Casals, P., Sarazin, M., "Perturbations to astronomical observations at the European Southern Observatorys very large telescope site in Paranal, Chile: analyses of climatological causes," Theor. Appl. Climatol., 73, 133-150 (2002)

[2] Boffin, H. M. J., Sedaghati, E., Blanchard, G., and 10 others, "Regaining the FORS: making optical groundbased transmission spectroscopy of exoplanets WASP-19b with VLT+FORS2," axXiv:1503.04155v2 (2015)

[3] Patat, F., "The Dancing Sky: 6 years of night sky observations at Cerro Paranal," arXiv:0801.2270v1 (2008)

[4] Kerber, F., Querel, R. R., Neureiter, B., Hanuschik, R., "Through thick and thin: Quantitative classification of photometric observing conditions on Paranal," Proc. SPIE 9910 (2016)

[5] Gilmozzi, R., Spyromilio, J., "The European Extremely Large Telescope (E-ELT)," The Messenger No 127 (March 2007)

[6] Sanders, G.H., "The Thirty Meter Telescope (TMT): An International Observatory," J. Astrophys. Astr., $34,81-86(2013)$

[7] Johns M., McCarthy, P. J., Raybould, K., Bouchez, A., Farahani, A., Filgueira, J. M., Jacoby, G., Shectman, S., Sheehan, M., "Giant Magellan Telescope: Overview," Proc. SPIE 8444, Ground-based and Airborne Telescopes IV, 84441H (17 September 2012)

[8] Rigaut, F., Neichel, B., Boccas, M., and 26 others, "Gemini multiconjugate adaptive optics system review I. Design, trade-offs and integration," Mon. Not. of the Royal Astron. Soc., 437, 2631-2375 (2013)

[9] Herriot, G., Hickson, P., Ellerbroek, B. L., and 8 others, "NFIRAOS: TMT facility adaptive optics with conventional DMs," Proc. SPIE 5903 Astronomical Adaptive Optics Systems and Applications II, 590302 (25 August 2005)

[10] Neichel B. and Rigaut F., "Rayleigh scattering, Fratricide effect and spot elongation: first on-sky results with GeMS," AO4ELT2, Victoria, Canada (2011)

[11] Otarola, A., Neichel, B., Wang, L., Boyer, C., Ellerbroek, B., Rigaut, F., "Analysis of fratricide effect observed with GeMS and its relevance for large aperture astronomical telescopes," AO4ELT3, Florence, Italy (2013)

[12] Wang, L., Otarola, A., Ellerbroek, B., "Impact of sodium laser guide star fratricide on MCAO systems," J. Opt. Soc. A. A, 27, A19-A28 (2010)

[13] Holzlöhner, R., "LGS Availability", 12th Annual Workshop on Laser Technology and Systems for Astronomical Adaptive Optics, Tenerife, Canary Islands, Spain, June 23-24 (2017)

[14] Winker, D. M., W. H. Hunt, and M. J. McGill, "Initial performance assessment of CALIOP," Geophys. Res. Lett., 34, L19803 (2007)

[15] Cho, H-M., Yang, P., Kattawar, G.W., Nasiri, S.L., Hu, Y., Minnis, P., Trepte, C., Winker, D., "Depolarization ratio and attenuated backscatter for nine cloud types: analyses based on collocated CALIPSO lidar and MODIS measurements," Optics Express, 16 (6), 3932-3948 (2008)

[16] Sassen, K., "The polarization lidar technique for cloud research: A review and current assessment," Bull. Am. Meteorol. Soc., 72, 1848-1866 (1991)

[17] Sassen, K., Benson, S., "A midlatitude cirrus cloud climatology from the facility for atmospheric remote sensing. Part II: Microphysical properties derived from lidar depolarization," J. Atmos. Sci., 58, 2103-2112 (2001)

[18] Nazaryan, H., McCormick, M.P., Menzel, W.P., "Global characterization of cirrus clouds using CALIPSO data," Journal of Geophysical Research, 113, D16211 (2008)

[19] Larroza, E.G., Nakaema, W.M., Bourayou, R., Hoareau, C., Landulfo, E., Keckhut., P., "Towards an automatic lidar cirrus cloud retrieval for climate studies," Atmospheric Measurement Techniques, 6, 3197$3210(2013)$

[20] Erasmus, D.A., and C.A. van Staden: "A Comparison of Satellite-Observed Cloud Cover and Water Vapor at Mauna Kea and Selected Sites in Northern Chile, the Southwestern U.S.A. and Northern Mexico. Final Report," New Initiatives Office, Aura Inc. 75pp (2003)

[21] della Valle, A., Maruccia, Y., Ortolani, S., Vitelli, V., "Analysis of the fraction of clear sky at the La Palma and Mount Graham sites," Mon. Not. R. Astron. Soc., 401, 1904-1926 (2010) 
[22] Garreaud, R.D., "The climate of northern Chile: mean state, variability and trends," Proc. Rev. Mex. De Astron. y Astrof., Serie de Conferencias 41, Ed. M. Cur, A. Otarola, J. Marin, M. Sarazin, 5-10 (2010)

[23] Wittet, D.C.B, Bode, M. F., Murdin, P., "The extinction properties of Saharan dust over La Palma," Vistas Astron., 30, 135-144 (1987)

[24] Sicard et al., "Results of site testing using an aerosol, backscatter lidar at the Roque de los Muchachos Observatory," Mon. Not. R. Astron. Soc. 405, 129142 (2010)

[25] Young, A., "Scintillation noise in CCD photometry," The Observatory, 113, 41-42 (1993)

[26] Boyer, C., et al., "Adaptive optics program at TMT," AO4ELT5, Puerto de La Cruz, Tenerife, Canary Islands, Spain (2017)

[27] Trubey, M., et al., "Thirty Meter Telescope Laser Guide Star Facility Design Update," AO4ELT5, Puerto de La Cruz, Tenerife, Canary Islands, Spain (2017)

[28] Seinfield, J.H., Pandis, S.N., "Atmospheric Chemistry and Physics: From air pollution to climate change," John Wiley \& Sons (1998)

[29] Buton, C., et al., "Atmospheric extinction properties above Maunakea from the nearby SuperNova factory spectro-photometric data set," Astronomy and Astrophysics, 549, A8 (2013)

[30] Pedreros, F., Holzlöhner, R., Budker, D., Lewis, S., Rochester, S., "Sodium vapor cell laser guide star experiments for continuous wave model validation", Proc. SPIE 9909, Adaptive optics Systems V, 99095P (27 July 2016)

[31] Pfrommer, T., Hickson, P., "High-resolution lidar observations of mesospheric sodium and implications for adaptive optics," J. Opt. Soc. Am. A, 27, A97-A105 (2010)

[32] Sivo, G., et al., "Getting ready for GeMS 2.0: A workhorse AO facility," AO4ELT5, Puerto de La Cruz, Tenerife, Canary Islands, Spain (2017) 